\title{
Market-Skilled Agents for Automating the Bandwidth Commerce
}

\author{
Monique Calisti ${ }^{1}$, Boi Faltings ${ }^{1}$, and Sandro Mazziotta ${ }^{2}$ \\ 1 Laboratoire d'Intelligence Artificielle \\ Swiss Federal Institute of Technology (EPFL) \\ CH-1015 Lausanne, Switzerland. \\ \{calisti,faltings\}@lia.di.epfl.ch \\ 2 Swisscom AG Corporate Technology \\ Ostermundigenstrasse 99 \\ CH-3050 Bern, Switzerland. \\ sandro.mazziotta@swisscom.com
}

\begin{abstract}
In the current deregulated Telecom scenario the number of network operators and service providers is rapidly increasing. The resulting competition generates the need for a flexible resource management. In parallel, the fast growth of electronic commerce opens a new market where operators can offer network bandwidth commodities. In this dynamic context, the traditional architecture of Telecom networks needs to be evolved. The integration of economic principles with software technology is a strategic contribution to this evolution. In particular, market-skilled software agents seem to be one of the most promising paradigms. In this paper we describe an agent-based system for the e-commerce of "IP bandwidth", and we discuss how the integration of this system within a network management platform would automate the advertisement of Telecom goods, the monitoring of market trends, the pricing and the configuration of network resources.
\end{abstract}

\section{Introduction}

The idea of deploying economic mechanisms for a variety of applications has been actively deployed in several fields. With the Internet providing a powerful and worldwide accessible medium for communicating and advertising business information, Internet-based commerce is flourishing. Virtual market-places facilitate in fact commerce transactions by bringing together buyers and sellers 8 . Many service and network providers are therefore considering, and in some cases already deploying 1 , electronic market places to offer services. These services include for instance fiber and satellite bandwidth, IP telephony minutes, etc. Even though this kind of business is more and more popular, the risk of failing or not being effective is not negligible. Several researchers believe that a smart generation of e-commerce would reduce these risks by integrating market-based

\footnotetext{
${ }^{1}$ As reported by Makris at least seven bandwidth brokers are already active on the Web [10].
} 
techniques within agent technology (see [14, 44 for good examples). Automated agents can follow more efficient and more flexible specific strategies, and can evaluate and optimise the utility of specific actions (such as bidding, offering, etc.). However, the integration of economic principles in electronic environments reveals the limitations of software instruments and the need to modify some economic mechanisms from their original formulation.

This paper reports the experience gained by developing a multi-agent auction house, the IP-market, for trading IP-bandwidth goods. The architecture of this auction house is not dependent on the specific type of goods offered in the market. For this reason, the lesson we report has a general validity that can be useful for the development of electronic market places selling a variety of goods. The novelty of our approach is in the automatic integration of an Intelligent Resource Manager (IRM) within the auction house on one side and within the network management platform on the other. While, some existing Internet-based auction houses allows human network operators to enter offers, our paradigm aims to automate the overall negotiation process. From the good offering phase to the end of trading transactions, software entities autonomously act on behalf of humans. This is possible because of the integration of the IRM within the network resources management and because of the agent communication structure supported by the IP-market. The description of the architecture of the auction house shows how the integration of this system within a network management platform has the potential of automating the advertisement of Telecom goods, the negotiation, the monitoring of market trends, the pricing and the configuration of network resources, as it is depicted in Figure 1. Another major contribution of this work is on the use of standard agent-to-agent communication facilities. This renders the IP-market open and very easy to access, which is essential to encourage several potential buyers and sellers to enter the electronic IP commerce. Finally, authors hope to stimulate and contribute to a productive discussion about the concrete challenges for the success of the e-commerce of Telecom services.

Section 2 gives some background on market-based approach to QoS and bandwidth allocation in the networking field and briefly reviews auctioning principles. Section [3] describes the IP-market simulator architecture. More focus on agent interactions and on the lessons learned is given in Section 3.4, with additional comments on the potential of integrating negotiating agents with a network management platform. Section 5 concludes the paper with final comments on the major contributions.

\section{Background}

There have been several efforts to exploit markets for distributed applications that need to coordinate a set of autonomous entities [9], 14]. The main motivations come from extensive studies done by economists about the problem of coordinating multiple agents in a societal structure [6]. The key advantage is that economics offers mechanisms that produce globally desirable results, avoid central coordination, and impose minimal communication requirements [18]. 


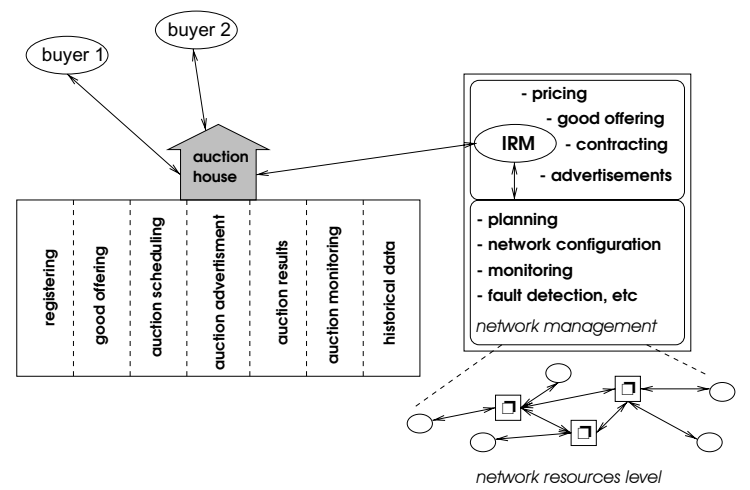

Fig. 1. The IRM integrates e-commerce with network management functionalities.

The auction model is a major paradigm which nowadays has been more and more deployed. In particular, many different types of auctions are appearing on the Internet, where electronic auction houses allow the sale of a large variety of products. These institutions are environments where certain kinds of goods are traded under explicit trading conventions. Gibney et al. are exploring the use of a two-levels auctioning mechanism for routing service demands in Telecom networks 9]. However, their main assumption is that a negotiation, namely a double auction, can only be started by a request coming from an end-user. Furthermore, the research is more focused on efficiently distributing the control of network resources, than on automating the potential of selling Telecom services through the use virtual market-places. Kubawara, et al. developed a market model for multi-agent resource allocation [7. In their market, Activity-Agents (the buyers) and Resource-Agents (the sellers) of the network resources trade bandwidth on physical ATM links, i.e., ATM channels. An 'equilibratory' approach is investigated: the market calculates the supply and demand volume equilibrium (no auctions take place). An interesting part of this work is the definition of sellers' and buyers' strategies. In this market model a fundamental assumption is that "sellers do not know the prices of the resources at other locations when they determine the prices". This is not always the case in a real market, but it is a realistic assumption for participants that first enter the market. Semret et al. deployed an auction-based pricing approach for differentiated Internet services [16] and they identified the best strategies for end-users and brokers. However, despite the very interesting conceptual results and the relative simulation evaluations, it is not easy to estimate if such mechanisms could be implemented in real networks.

In the MACH project 2 , we developed the IP-market simulator, a virtual market place for selling IP bandwidth with given Quality of Service, QoS. In this environment, IP Network Operators are the sellers and all potential customers, i.e., final end-users, IP brokers and all kind of Value Added Service Providers

\footnotetext{
${ }^{2}$ More information about MACH, MArket meCHanism for selling on-demand IP bandwidth, are available at: http://liawww.epfl.ch/ calisti/MACH
} 
(voice, video, multimedia), are the buyers. The integration of seller agents within the underlying network infrastructure eliminates the need of human intervention for offering goods to a virtual market-place and allows the automation of network resources' negotiation and pricing. Furthermore, the use of a standard agent communication language and of standard interaction protocols guarantee an open and accessible auction house.

\subsection{Electronic Auctions}

The concept of auction is very common and almost everybody is familiar with this trading paradigm, but the simplicity is only superficial. Smart auction principles and bidding strategies have being analysed and studied in various ways, in order to maximise the revenue. The work ranges from more theoretical and general principles [2], to very specific studies related to auctions for selling specific products, such as wine and art 1], second hand automobiles [11, etc. Electronic auctions are becoming more and more popular due to basically three main factors. First, they represent an alternative way to implement negotiation and to establish prices. Second, auction mechanisms are more and more known: deep studies of how to optimise auction protocols and strategies promise efficient results [14]. Finally, the success of the Internet and the development of the e-commerce give to auctions a natural environment, where a larger number of potential customers and suppliers can settle business transactions in a shorter time.

There are qualitatively different auction settings depending on how bidders evaluate a good. In private auctions the evaluation is not dependent on external factors and is based on private bidder's information. Auctions are non-private if the valuation of bids is decided upon private considerations and valuations of others' bids. If the value is partly dependent on other's values the auction is called correlated value auction. Whether the value is completely dependent on other's bid valuations the process is called a common value auction. Considering the IP-bandwidth market, the auction setting depends on whether the bidder is a final end-user or a potential re-seller. Auction protocols establish the order in which prices are quoted and the manner in which bids are tendered. There are basically 4 major one-sided auction protocols: English, First-price sealed-bid, Vickrey and Dutch. A good list of references is given in 15] and good reviews are given in [8] and [14. The IP-market uses the first three types of protocols. Several considerations are important when defining an auction strategy: first, a seller has to choose an auction protocol, which means he has to predict the behaviour of bidders. A buyer makes an estimation of his own value of the good and evaluates what other bidders are likely offer. A seller can influence auction results by revealing information about the good. An optimal strategy from a seller's perspective is to reveal information, since in general the more information a bidder has, the more a price moderation effect of winner's curse is reduced [14]. The revenue-equivalence theorem [12] demonstrates that, under the assumption of private value, all four basic auction types produce the same expected value to the auctioneer when bidders are risk-neutral and symmetric. 
However, this theoretical results do not apply in many real situations. From the bidder's perspective the kind of auction protocol has the effect of revealing more or less information in the course of the auction. Bidders must decide the maximum amount they will bid, based on their own valuation of the good and their prior beliefs about the valuations of other bidders. If useful information is revealed during the auction, bidding strategies can take into account this additional input. In the IP-market a negotiation strategy is coded into agents using a set of rules specifying how and when they should bargain and/or accept a deal (rule-based approach).

\section{The IP-Market Architecture}

In the IP-market it is not a network operator that directly offers IP bandwidth goods, but an intermediary, the auctioneer, who calls for tenders and adjudicates the purchase to the highest bid. The IP-market is designed to be fair and neutral in respect to both sellers and buyers: it is a third trusted party that guarantees standard and known trading mechanisms to all participants in the same way.

In order to cast the IP bandwidth commerce in terms of a computational market, it is necessary to specify: the goods traded, the buyer and the seller agents' characteristics and the markets structure.

\subsection{Modelling the Goods}

At a very abstract level, the type of good offered in our market is a certain amount of bandwidth with some QoS guarantees. Referring to the Differentiated Service framework [5], the complexity of QoS specification is abstracted by three levels: gold, silver and bronze. Gold stands for virtual leased lines, silver for better than best-effort and bronze symbolises the classical best-effort. Different entities in the market can have different internal representations of the market's goods, however, for a common global understanding, a minimal agreement on a common model is needed. This implies the need of abstracting from specific internal representations and of defining a common ontology to refer to. The common representation of the good expresses an agreement between the buyer and the seller that the service which the customer bids for corresponds to what the seller is committing to provide. This is formalised by the Service Level Agreements (SLAs [17]). Since SLAs depend on the nature of services provided by a network operator, and since we are far from having a standard and common way of expressing SLAs, the big challenge for software developers willing to implement agents reasoning about SLAs, is how to represent them (the SLA representation is not covered in this paper). In the following we describe the good's representation that is valid in the IP-market .

Network provider's good representation. The IP network has been modelled as a set of nodes interconnected by links. The nodes represent routers and/or switches in the network and the links represent connections existing between nodes. 
Every link can be characterised by several parameters, such as price, available bandwidth, end-to-end delay, etc. Figure 2 shows two different levels of abstractions: link-level (e.g., $a-a 1$ ) and path-level (e.g., $a-e)$.

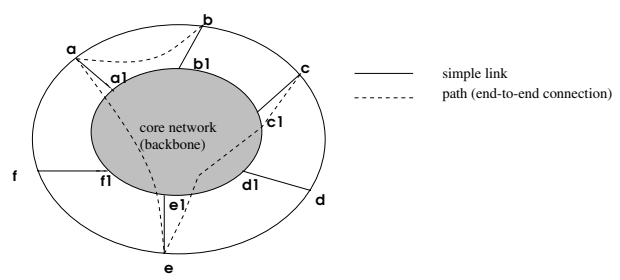

Fig. 2. The abstract network model.

Market's good representation. Every good offered to the IP-market must be described in terms of: end-points, i.e., termination points of the end-to-end path, asking-price that is the starting price of the good, resale-price that expresses the demand for the good on the IP-market, i.e., the value at which the good could be eventually re-sold. This value is fixed for the duration of an auction, is known before the auction starts, it can be defined according to the market history or it may be defined in terms of external supply considerations. The resale price can be either set by the seller, or defined as a function of other market-variables or set according to other conventions. The offer timeout is the time the offer will be valid for and time states when the service will start and stop to be available. The amount of bandwidth and the QoS level, i.e., bronze, silver or gold, characterise the connection type.

The buyer's view of the goods is built upon the information that the IPmarket makes publicly available, which are usually the same described above.

\subsection{The Market Actors}

Several entities populate the IP-market. Seller Agents (SA) represent network and/or service providers and send IP-bandwidth offers to the market house. Buyer or Bidder Agents (BA) represent all potential customers and send bids to the market in order to purchase a specific good. For every auction process that takes place in the market, an auctioneer agent (AA) is responsible to manage it. An AA receives bids from buyers, evaluates them and determines the winner. At the end of an auction an AA notifies both the winner and the seller about the results of the transaction. Two different kinds of potential buyers can access the IP-market: end-customers or final and re-sellers customers, or non-final. A private value auction model is valid for end-customers that are willing to buy IP-bandwidth for private usage and do not intend to re-sell the IP-good to the market. Re-seller or broker customers are willing to re-sell the IP-bandwidth to other end-customers. In this last case the bidder's value of a good depends partly on its own preferences and partly on others' values, i.e., non-private value settings. For this reason, under the same auction protocol end-customers and re-sellers may deploy different strategies. 


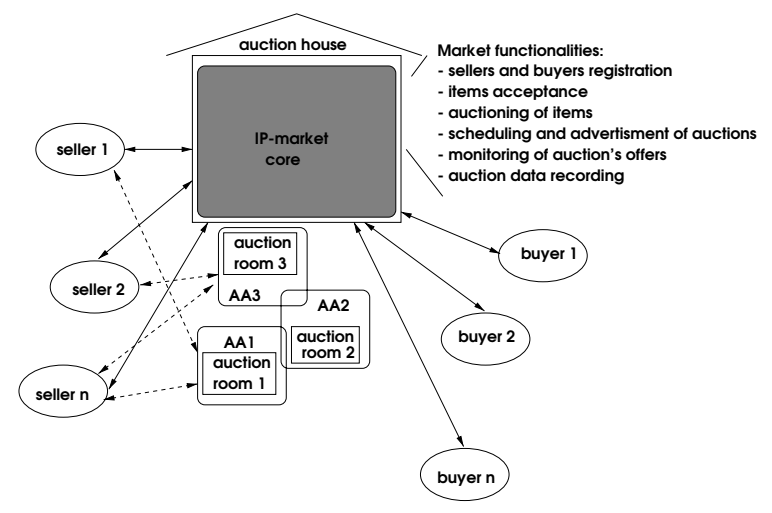

Fig. 3. The IP-market architecture. Buyer and seller agents directly interact with the IP-market core. Buyers participate in specific auctions by interacting with an Auctioneer Agent in an auction room.

Every BA is assumed to know the preferences of the user is acting on behalf of. The set of preferences is specified at the creation of every BA, so that a user profile is generated. Whenever an auction is started, a BA evaluates whether to take part in the auction or not by comparing the user profile and the goods' characteristics. If the $\mathrm{BA}$ enters a specific auction, the bidding strategy will be based on a set of rules that take into account the user profile, other user's preferences parameters, when available, and historical data about past auctions. The BA is also responsible for dynamic updates of the user profile.

\subsection{The IP-Market Core}

The IP-Market core is the central brain of the overall simulator, see Figure 3. by supplying the mechanisms required for managing individual auction process and for coordinating parallel auctions with other market activities.

In the IP-market simulator several auctions can take place in parallel. Every auction process runs in a separate virtual auction room managed by a different AA. Dedicated entities for managing distinct auctions increase the scalability and the efficiency of the market. All communications concerning a specific auction process are handled transparently to all other market activities. While an auction is going on, the market can for instance accept new incoming goods, register new buyers, etc. In order to access the market-place and to be able to participate to trading transactions, seller and buyer agents need to register within the market. A registered SA can then offer items to the market, and a registered BA can then participate in one or more auctions. The identity of every auction's participants is kept confidential. The auctioneer determines the winner and notifies participants about the auction results. After this notification, the winner and the seller can directly contact each other in order to fix the contract details 3 . More precisely, the core functionalities offered by the IP-market are:

\footnotetext{
3 The current IP-market implementation does not support automatic contract definition routines.
} 
- Registration of incoming sellers and buyers for identifying all potential trading participants. The market creates and records a profile for each registered entity that is then used for controlling market's access and trading activities. At the registration, every agent has to declare the name of the seller or of the buyer that is acting on behalf of, an address, an e-mail, a password that will be used to verify future accesses, a phone number, etc.

- Registration of IP-bandwidth offers. During this phase, a registered seller offers a specific item o the IP-market . The IP-market creates an auctioneer agent and informs the seller about the auctioneer agent's name and the auction identifier.

- Scheduling auctions and notification of potential bidders (i.e., all registered buyers) about the current active auctions. Different mechanisms can be used for auction scheduling: regular schedule when fixed appointments are established during the day, and asynchronous schedule when the good is made available (an auction is activated) as soon as the good registration succeeds. Registered buyers can either receive advertisements from the market, or proactively ask which auctions are currently active.

- Displaying all active auctions, registered buyers and sellers.

- Creation of auctioneer agents, one for every auction that is activated. In order to deploy historical results, every auctioneer records auction's data that the market will maintain and manage. From these data structures it is possible to extract information that agents will use for future speculations.

- Routing of all messages to and from agents that have been registered with the market.

- Winner computation and notification of all auction participants about the final results (these tasks are performed by the auctioneer agent). If a single item is sold within every auction, the winner computation is not a very difficult task (it takes $O(\mathrm{am})$ where a is the number of bidders and $\mathrm{m}$ is the number of items sold per auction). If multiple items are offered during the same auction process the complexity of the winner determination increases. If the goal is to maximise the revenue the optimal winner determination becomes NP-complete [13. The combinatorial auction infrastructure is currently under construction.

- Closing auction phase. The auction is successful when a winner has been selected and the bid is compliant to the seller requirements. Both winner and seller are expected to confirm that they accept auction's results.

Security issues represent a common open problem for many e-commerce applications, that is even more crucial for agent-based systems, where distributed and autonomous software entities increase the number of possible attacks points. The current IP-market implementation needs more work on security aspects: while a login-name and a password can be enough for a simulator, a real market would need to verify the truthfulness of the information supplied by buyers and sellers. On the other hand, buyers and sellers would need to verify the market's behaviour and the fairness of auctioneer agents. For a real implementation specific agreements among all potential participants would be required. 


\subsection{An Open and Inter-operable Market}

A preliminary and fundamental feature of an open and inter-operable marketplace is the capability of supporting standard communications. Two main issues need to be solved: messages have to be physically delivered to the right destination and delivered messages need to be understood.

The first task, common to all distributed systems, requires that the IP-market is capable of addressing and routing messages to remote and distributed entities. For this purpose, every agent has a unique identifier and a unique address that the Agent Communication Router, (ACR) processes to forward messages. The ACR is part of the IP-market and is a sub-component devoted to message routing. Once a message has been delivered, full inter-operability can be reached only if the message can be conveniently processed. Figure 4 visualises different levels of understanding. This is first reached through the use of standard agent interaction protocols, which means standard sequences of messages. For every communication phase, i.e., starting auction, bidding, closing auction, etc., a specific protocol is selected. Some standard FIPA protocols have been adopted and some new ones have been defined. FIPA [3] is a non-profit standardisation group that aims to promote inter-operability of emerging agent-based applications, service and equipment. In particular FIPA defined standard communication facilities. Inter-operability is also achieved by the usage of a common

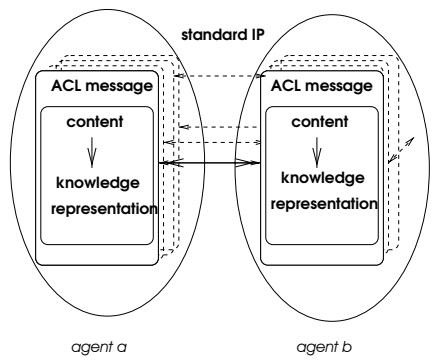

Fig. 4. In order to achieve full inter-operability a common understanding is needed at different level: standard interaction protocols, a common ACL, a common content representation and the same ontology.

agent communication language, which allows to parse and process delivered messages in a standard and common way. The official market-place language is FIPA ACL 3]. The first element of an ACL message is the name of the performative, the following part is a sequence of parameters that can occur in any order in the message. In particular the :protocol field specifies the expected sequence of messages during a specific agents' conversation. The :content field of a refers to whatever the communicative acts apply to. In the IP-market an ad hoc common content language capable to express actions, objects, and propositions has been developed and deployed. An action is an activity that can be performed by some agent, e.g., activate-auction. An object in the language defines an identifiable thing (with a name), e.g., a good. A proposition states that a sentence in a language is true or false, e.g., auction-successful. By convention, agents accessing 
the IP-market use this common language. Finally, a common ontology has been referred. An ontology is an explicit specification of the structure of a certain domain. This includes a vocabulary for referring to the subject area, and a set of logical statements expressing the constraints existing in the domain and restricting the implementation of the vocabulary. The IP-market ontology provides a vocabulary for representing and communicating knowledge about IP-bandwidth goods and about auctioning mechanisms. Furthermore, a set of relationships and properties that hold for the entities denoted by this vocabulary has been fixed.

The IP-market and all its auxiliary components, such as ACL parsers, ACR, bidder, seller and auctioneer agents, etc. have been implemented in Java. Successful tests have proved the inter-operability between the IP-market, developed at the LIA Laboratory in Lausanne, and seller agents developed by Swisscom. In particular, seller agents running in machines located in Bern (at Swisscom), have successfully registered with the IP-market running remotely at in Lausanne (at LIA). The connection has been established through the use of Java messages over TCP-IP sockets. Swisscom seller agents offered IP-services between different cities in Switzerland with different amount of available bandwidth and various QoS characteristics. Buyers agents, previously created and registered with the IP-market, registered for active auctions matching their preferences. The predesigned and standard interaction protocols and the common terms used during all agent conversations have guaranteed full interconnectivity, so that auctions have successfully been run.

\section{Integrating E-Commerce Capabilities within a Network}

The Intelligent Resource Manager (IRM) automates all interactions between the IP-market and the network infrastructure. The interaction with the network management platform on one side and with the IP-market on the other side allows the integration of the e-commerce activity with traditional network management functionalities such as planning, configuration, resource allocation, monitoring, etc. This kind of architecture leads to advantages for both customers and providers. There are advantages for customers, since there is a third trusted party controlling all trading transactions: the IP-market auction house supplies in fact standard and fair mechanisms for all potential participants. The providers' advantages come from the fact that such an architecture would:

- Automate the advertisement of Telecom and/or Internet goods, according to network resources availability.

- Accelerate various steps of the service provisioning process

- Provide an alternative way to sell bandwidth surplus

- Support an automatic pricing mechanism through the auction paradigm.

The IP-market is open and inter-operable since standard communication techniques have been adopted. The common ontology that has been defined and deployed contains terms and definitions for the e-commerce of IP-goods. This ontology can be enriched and eventually modified to enable agent negotiations 
to address more specific markets, such as IP telephony, video on demand, etc. Furthermore, agents can simply refer to a different ontology in order to deploy the same IP-market structure for the e-commerce of books, flowers, wine or any other kind of good.

Flexibility is given by IP-market structure that is able to support transactions independently on the type of good that is offered and on the type of trading paradigm that is deployed. The IP-market can currently run auctions, but it is possible to integrate supports for other trading paradigms, such as automatic contract-net mechanisms . Furthermore, focusing on auctioning capabilities, several protocols are available: Vickrey, English and First-Price Sealed-Bid. In addition, for auction scheduling two different mechanisms have been implemented: regular and asynchronous. The auction advertisement can be either automatic, i.e., once an agent register with the market a notification of active auctions is received, or pro-actively triggered by an agent request.

Scalability is mainly achieved thanks to the modular structure of the market, which makes it possible to satisfy an high number of participants. Different market's sub-modules are in fact devoted to solve specific tasks such us users' registration, message routing, auctions' advertisement, etc. Furthermore, the creation of dedicated auctioneers for every distinct auction process allows more efficient management of agent communications and auction timing thereby increasing the potential scalability.

\section{Conclusion}

This paper reports the experience learnt during the implementation of the IPmarket, an electronic auction house for selling 'IP-bandwidth goods'. Particular attention was devoted to concrete issues faced for developing an open, interoperable, flexible and scalable virtual market.

The authors believe that the major contribution of this work is in showing the feasibility of an automated interaction between buyers and sellers for the e-commerce of Telecom services. Furthermore, it shows the potential of deploying auctions for pricing IP services. Secondly, as stated above, the deployment of standard agent interaction instruments enables this system to be open and easy to access. Finally, the IP-market formalism abstracts networking complexity by providing a 'customer-friendly' view of IP end-to-end connections with QoS guarantees. Future work on the IP-market simulator includes transferring the platform over the Web, enhancing the system with XML-based descriptions of the goods traded, and integrating multi-items auctions facilities.

Acknowledgements. Many thanks to the other participants of the MACH project, especially to Sankalp Upadhyay and Thomas Fayet for their fundamental work and to Steven Willmott for his precious suggestions. 


\section{References}

1. O. Ashenfelter, "How auction work for wine and art". Journal of Economic Perspectives, Vol. 3, No. 3 (Summer 1989) pp. 23-36.

2. R. Cassady, "Auction and Auctioneering". Reading, Univ. California Press, Ont. 1979, ISBN 0520002164.

3. FIPA'97 Specifications. Available on line at: http://www.fipa.org

4. R. Guttman, A. G. Moukas and P. Maes, "Agent-mediated Electronic Commerce: A Survey". Knowledge Engineering Review, 1998.

5. T. Li and Y. Rekhter. "RFC 2430: RA Provider Architecture for Differentiated Services and Traffic Engineering" (PASTE), October 1998.

6. T.C. Koopmans, "Uses of prices". Scientific Papers of Tjalling C. Koopmans, Springer-Verlag, 1970. pp. 243-257.

7. K. Kuwabara, T. Ishida, Y. Nishibe and T. Suda, "An Equilibratory Market-Based approach for distributed resource allocation and its application to communication Network Control". In Market-Based Control, Worl Scientific, Editor Scott H. Clearwater, 1996.

8. M. Kumar and S. I. Feldman, "Internet Auctions". Technical report, November 1998. http://www.ibm.com/iac/tech-paper.html

9. Gibney M.A. and Jennings N.R., "Dynamic Resource Allocation by Market-Based Routing in Telecommunications Networks". In Proceedings of the 2nd International Workshop on Intelligent Agents for Telecommunication Applications (IATA'98), LNAI, Vol. 1437, pp. 102-117, Springer, July 4-7 1998.

10. J. Makris, "Not Exactly Nasdaq". http://www.eocenter.com/internetwk/

11. Lee, H.G., "Do electronic marketplaces lower the price of goods?", Comm. of the ACM, Vol. 41 No. 1, Jan. 1998, pp. 73-80.

12. A. Mas-Colell, M. Whinston, J.,R. Green, "Microeconomic Theory". Oxford University Press, 1995.

13. T. Sandholm, "An Algorithm for Optimal Winner Determination in Combinatorial Auctions". In Proceedings of the 16th International Joint Conference on Artificial Intelligence (IJCAI'99), Stockholm, Sweden, 1999.

14. T. Sandholm, "Automated Negotiation". Communications of the ACM 42(3), 8485, 1999. Special issue on Agents in E-commerce.

15. T. Sandholm, "Issues in Computational Vickrey Auctions". International Journal of Electronic Commerce, 1999.

16. Semret, Liao, Campbell, Lazar, "Market Pricing of Differentiated Internet Services", 7th IWQoS, London, May 31 - June 4, 1999.

17. D. Verma. "Supporting Service Level Agreements on IP Networks". Macmillan Technical Publisher, September 1999.

18. M.P. Wellmann, "A computational market model for distributed configuration design". In Proceedings of 12th conference of the American Association on Artificial Intelligence (AAAI'94), p. 401-409, Seattle, WA, 1994. 